\title{
Serum liver enzyme profile in Timor deer (Cervus timorensis) with fascioliasis in Indonesia
}

\author{
Purnama, M.T.E. ${ }^{1}$, Dewi, W.K. ${ }^{2}$, Triana, N.M. ${ }^{2}$, Ooi, H.K. ${ }^{3 *}$ \\ ${ }^{1}$ Department of Veterinary Anatomy, Faculty of Veterinary Medicine, Universitas Airlangga, Mulyorejo Campus C Surabaya, East Java 60115, Indonesia \\ ${ }^{2}$ Laboratory of Anatomy and Clinical Pathology, Faculty of Veterinary Medicine, Universitas Airlangga, Mulyorejo Campus C Surabaya, East Java 60115, \\ Indonesia \\ ${ }^{3}$ Department of Veterinary Parasitology, School of Veterinary Medicine, Azabu University, 1-17-71, Fuchinobe, Sagamihara, Kanagawa 252-5201, \\ Japan \\ *Corresponding author: hkooi@azabu-u.ac.jp
}

\section{ARTICLE HISTORY}

Received: 24 August 2020

Revised: 27 November 2020

Accepted: 1 December 2020

Published: 25 March 2021

\begin{abstract}
Timor deer (Cervus timorensis) at Surabaya zoo, Indonesia, that were found to be naturally infected with Fasciola, showed elevated level of serum alanine aminotransferase (ALT), aspartate aminotransferase (AST), and alkaline phosphatase (ALP). Of a total of 75 deer examined, $12(25 \%)$ of the 47 adult deer and $8(29 \%)$ of the 28 juvenile deer were found to be infected with fascioliasis, as evidenced by the shedding of the parasite eggs. The level of ALT, AST and ALP were significantly elevated $(p<0.05)$ in all the infected deer. Only Fasciolainfected deer showed elevated serum liver enzyme. Deer with elevated enzyme level show a trend that positively correspond with higher Egg per gram of feces (EPG). The average size of the parasite eggs at $169.0 \pm 11.1 \times 96.0 \pm 3.5 \mu \mathrm{m}$, correspond well with that of Fasciola gigantica. No other trematode eggs were observed besides that of $F$. gigantica. There was no significant difference in the enzyme profile between the two sexes in both the infected and the uninfected group. This is the first report of the elevation of serum liver enzyme in Timor deer that is associated with not only fascioliasis and also correspond positively with the EPG.
\end{abstract}

Keywords: ALT, AST, ALP, Fascioliasis, Serum liver enzyme, Timor deer.

\section{INTRODUCTION}

Timor deer (Cervus timorensis) are native animals of Indonesia and their range of distribution stretch from Java and Bali to the islands east of the Wallace line (Martins et al., 2018). In the West Bali National Park, a herd of the deer consisting of $72 \%$ adults and $28 \%$ juvenile, was observed to spend most of their daily routine foraging for grass and other plants in the savannah and seasonal forests (Masyud et al., 2007). The vegetation in these areas provides a good opportunity for the deer to be infected with digenetic trematode because of the abundance of snails.

Lymnaeid snails are known to serve as the intermediate host of Fasciola, which is a ruminant liver fluke. Fascioliasis is a parasitic disease that infects the liver and biliary duct of most ruminants. Under optimal condition, cercariae migrated out from the infected snail to attach themselves to the vegetation and then transform into metacercariae, which are infective to the ruminants. These metacercariae are then orally eaten together with the plants or grass to which they are attached to, by the deer (Robinson \& Dalton, 2009). Once ingested by the deer, the metacercariae develop to become immature worms, which penetrate through the intestinal wall into the peritoneal cavity where they find their way onto the liver surface. These immature worms then burrow into the liver parenchyma to reach the biliary duct where they develop into adult worm. In chronic fascioliasis in cattle, it had been reported that hepatocytes release aspartate aminotransferase (AST) when the immature worms penetrate the liver parenchyma (Kowalczyk et al., 2018). Hepatocellular necrosis and degenerative changes caused by the migration of immature worms through the sheep liver parenchyma are also associated with increased alanine aminotransferase (ALT) and alkaline phosphatase (ALP) (Hodzić et al., 2013). The general effect seen in all adult Fasciola-infected hosts is the alteration of the hepatic serum enzymes.

Fascioliasis in human resulting in various symptoms such as biliary colic, fever, bile duct obstruction and cholangitis, and acute pancreatitis had been reported (Ozturhan et al., 2009; Ozel et al., 2013). Immature worms that failed to migrate to the liver can produce abscesses in many locations and ectopic masses from degenerated hepatocyte cells and gallbladder cells can also formed (Sezgin et al., 2004).

Increase in serum level of ALT, AST and ALP can be indicators of hepatobiliary and hepatic cholestasis disease 
(Park et al., 2000). In the present study, serum concentration of ALT, AST and ALP of Timor deer were examined with reference to their infection with fascioliasis.

\section{MATERIALS AND METHODS}

\section{Timor deer}

Our study was conducted at Surabaya Zoo $\left(7^{\circ} 17^{\prime} 44^{\prime \prime S}\right.$, $\left.112^{\circ} 44^{\prime} 11^{\prime \prime E}\right)$, Surabaya, East Java, Indonesia, which has a total population of 293 Timor deer. These deer, of different age and sex, were kept in a fenced enclosure and fed chopped Pennisetum purpureum twice a day. Fresh water was available ad libitum in a pond within the enclosure.

Juvenile deer are generally defined as those male deer that are less than 2 years old but for the female deer, they become adult at the age of 15 months. Besides considering the size of the animal and the antler developmental stage, age determination was also supported by observing the four incisors in the male and female juvenile deer, that are replaced by permanent teeth at around the age of 12 months. The oldest deer in the zoo was 15 years old.

No anthelmintic treatment was given to the deer for the past year.

Based on Slovin's formula, 75 deer were required for the investigation to obtain the number of samples with a standard error of $10 \%$ (Tejada \& Punzalan, 2012). A total of 75 Timor deer in the zoo were randomly selected and herded into individual cages for taking the blood and fecal samples. The individual cages were placed in a dark room and the deer were handled by the animal keeper to minimize the stress level.

\section{Ethical approval}

This study was approved by the Institutional Animal Care and Use Committee, Faculty of Veterinary Medicine, Universitas Airlangga, with reference No: 689-KE. The approval came with the conditions that there should be no animal abuse and stress to the animals should be minimized as well as the number of samples collected be kept to a minimum.

\section{Collection and processing of blood serum and fecal samples}

Five $\mathrm{ml}$ of blood from the jugular vein were collected in a plain serum tube or Vacutainer ${ }^{\circledR}$ (BD, USA) and allowed to clot for 10-20 minutes in icebox, followed by centrifugation in a centrifuge machine Hettich EBA $200^{\circ}$ (Hettich, GER), at a speed of $2500 \mathrm{rpm}$ for 10 minutes or equivalent to $800 \mathrm{xg}$. The supernatant was used for measuring the serum level of ALT, AST, and ALP using the clinical chemistry analyzer Hitachi $902^{\circ}$ (Roche Diagnostics, USA).

After collecting their blood, fecal sample of the same animal was collected from their rectum with plastic bags. To detect the parasite eggs using a sedimentation method, 3 grams of feces were dissolved in $30 \mathrm{ml}$ of distilled water and stirred until a homogeneous solution was obtained. It was then filtered through 2 layers of cotton gauze (with a hole of $1 \mathrm{~mm} \times 1 \mathrm{~mm}$ ) and allowed to stand. The supernatant was removed slowly leaving the sediment. More distilled water was added to resuspend the sediment and the supernatant was decanted. This was repeated 3 times. Finally, all the sediment was placed onto the glass slide and observed under a light microscope. Samples that were positive for Fasciola eggs were then used for counting the number of Fasciola eggs per gram of the feces (EPG) using the modified McMaster chamber method as proposed by Conceição et al. (2002). Briefly, the first step distilled water was replaced with a solution of $5 \%$ sodium dodecyl sulfate (SDS) and 30 grams of faeces was examined. The sedimentation procedure was carried out as described above. After the last sedimentation and decantation, the sediment was transferred into a test tube and filled up to 50 $\mathrm{ml}$ volume with $5 \%$ SDS solution. The tube was agitated to re-suspend the sediment in the solution which was then placed into both chambers of a McMaster slide. All of Fasciola eggs present at the bottom of the McMaster chambers were counted and then the EPG was calculated.

\section{Statistical analysis}

The serum liver enzymes of the Timor deer were expressed as mean \pm standard deviation (SD). Significant differences in blood and fecal parameters between the uninfected and infected deer from each sex and age group were determined by Multivariate Analysis of Variance (MANOVA). The correlation between total EPG and serum liver enzyme levels was analyzed using Pearson's correlation test. The probability value of $p<0.05$ was considered as significant. All the data were analyzed using the SPSS Statistical Package software v.21 for Windows (IBM Corp., New York, USA) (Warne, 2014).

\section{RESULTS AND DISCUSSION}

Of a total of 75 deer examined, $12(25 \%)$ of the 47 adult deer and $8(29 \%)$ of the 28 juvenile deer were found to be infected with fascioliasis, as evidenced by the shedding of the parasite eggs. The profiles of the serum liver enzymes, ALT, AST and ALP, of both infected and uninfected juvenile and adult Timor deer, as well as their gender, are shown in Table 1. The level of ALT, AST and ALP significantly $(p<0.05)$ increased in all the deer infected with fascioliasis. However, no significant difference in the enzyme profile was observed between the two sexes within both the infected and the uninfected group.

The average measurement of a total of 63 eggs from 5 infected deer was found to be $169.0 \pm 11.1 \times 96.0 \pm 3.5 \mu \mathrm{m}$, which corresponds well with that of Fasciola gigantica (Valero et al., 2009). This showed that the deer were probably infected with only F. gigantica. No other trematode eggs besides that of $F$. gigantica, were observed. The feces of 2 adult deer were found to contain trichostrongylid eggs. The increase in the enzyme level of the individual deer as positively correlated with their EPG for the juvenile and adult deer are shown in Figure 1 and Figure 2, respectively. The range of the EPG among the deer was 0 to 1,450 .

Edith et al. (2010), in their experimental infection of riverine buffaloes with $F$. gigantica, reported that during the prepatent period of fascioliasis, a significant increase in the liver-specific enzymes AST and ALT in the host circulation was observed when the fluke start to invade into the liver causing traumatic hepatitis and also when the immature fluke start to develop into adult worm. This was well correlated with the increase of AST at 4 week- postinfection (WPI), ALT at $6 \mathrm{WPI}$ and ALP at $10 \mathrm{WPI}$, respectively. This report supports our observation in Timor deer that fascioliasis led to an increase in these serum liver enzyme.

Only those deer that were shedding the Fasciola eggs showed elevated serum liver enzymes, despite that both the infected and the uninfected deer were kept under the same condition and given the same feed. ALP is secreted through the bile duct and increase during cholestatic disease. Increased ALP synthesis by bile epithelial cells may lead to more ALP entering the blood stream. It is known that the amphoteric property of bile salts facilitate the release 
Table 1. Serum liver enzymes of different gender and age of Timor deer that were uninfected and infected with Fasciola gigantica

\begin{tabular}{|c|c|c|c|c|c|}
\hline \multirow{2}{*}{\multicolumn{2}{|c|}{ Group }} & \multirow{2}{*}{$\mathrm{n}$} & \multicolumn{3}{|c|}{ Profile values (mean $\pm S D$ ) } \\
\hline & & & $\operatorname{ALT}(\mu / L)$ & AST $(\mu / L)$ & $\operatorname{ALP}(\mu / L)$ \\
\hline \multicolumn{6}{|l|}{ Age $(n=75)$} \\
\hline Juvenile $(n=28)$ & Uninfected & 20 & $69.87^{a} \pm 6.42$ & $68.92^{\mathrm{a}} \pm 5.75$ & $158.84^{\mathrm{a}} \pm 4.64$ \\
\hline \multirow{2}{*}{ Adult $(n=47)$} & Uninfected & 35 & $74.09^{a} \pm 4.55$ & $52.16^{a} \pm 6.92$ & $138.91^{\mathrm{a}} \pm 19.90$ \\
\hline & Infected & 12 & $191.17^{b} \pm 44.89$ & $170.81^{b} \pm 21.09$ & $268.01^{b} \pm 51.54$ \\
\hline \multicolumn{6}{|l|}{ Gender $(n=75)$} \\
\hline \multirow[t]{2}{*}{ Male $(n=42)$} & Uninfected & 32 & $73.18^{\mathrm{a}} \pm 4.48$ & $58.70^{\mathrm{a}} \pm 10.98$ & $152.97^{a} \pm 10.40$ \\
\hline & Infected & 10 & $185.21^{b} \pm 42.99$ & $179.11^{b} \pm 18.21$ & $267.05^{b} \pm 48.29$ \\
\hline \multirow[t]{2}{*}{ Female $(n=33)$} & Uninfected & 23 & $71.70^{\mathrm{a}} \pm 6.94$ & $57.64^{a} \pm 9.72$ & $136.67^{a} \pm 23.37$ \\
\hline & Infected & 10 & $182.58^{\mathrm{b}} \pm 35.58$ & $170.28^{b} \pm 37.01$ & $266.77^{b} \pm 46.22$ \\
\hline
\end{tabular}

a,b Different superscripts in the same column indicate significant differences $(p<0.05)$.

ALT=Alanine Aminotransferase, AST=Aspartate Aminotransferase, ALP=Alkaline Phosphatase, $n=$ number of samples.

(A)

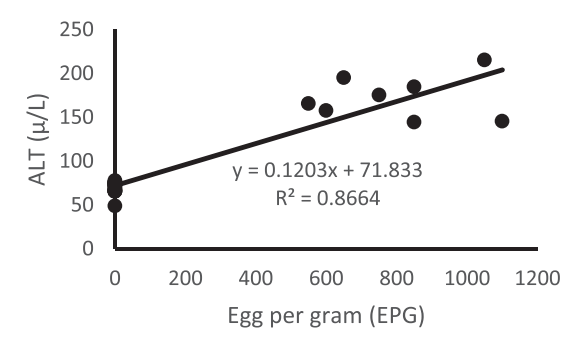

(B)

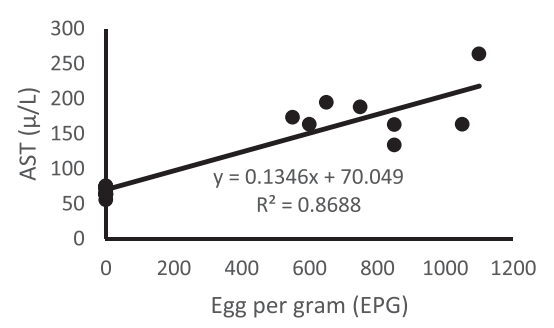

(C)

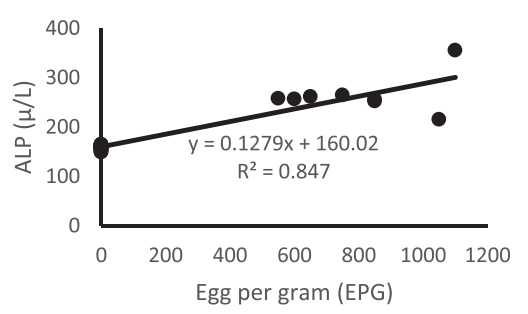

Figure 1. Correlation of serum liver enzyme values to Fasciola egg per gram (EPG) in juvenile Timor deer. (A) Correlation of ALT to EPG, (B) Correlation of AST to EPG, (C) Correlation of ALP to EPG.

(D)

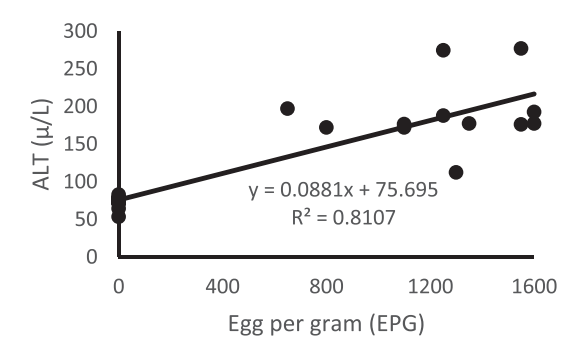

(E)

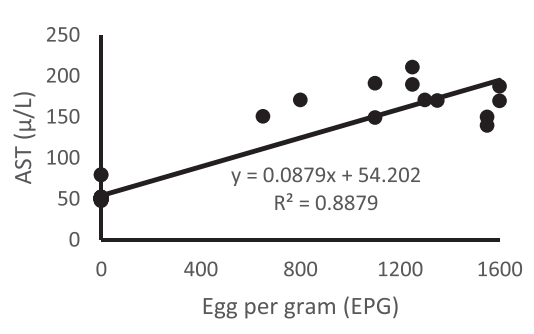

(F)

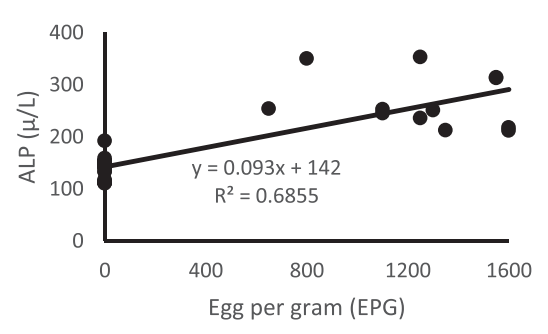

Figure 2. Correlation of serum liver enzyme values to Fasciola egg per gram (EPG) in adult Timor deer. (D) Correlation of ALT to EPG, (E) Correlation of AST to EPG, (F) Correlation of ALP to EPG.

of ALP from the cellular membrane into the blood (Hyder et al., 2013). Blockage of bile duct will also lead to the increase of ALP in the blood.

The AST enzyme is found mainly in the liver and heart muscle, and also at moderate concentrations in the skeletal muscles, kidneys, and pancreas. On the contrary, ALT is present in large quantities on the liver cell. Destruction of the liver cell or inflammation of the liver leads to a conspicuous increase in the concentration of these enzymes in the serum. When damage to the hepatic cell occurred, the release of ALT into the blood from the liver had been observed to last longer than the release of AST (Senior, 2012). Fasciola infection can manifest the acute phase and the chronic phase (Tolan Jr, 2011). The metacercaria phase will persist in the intestine until it becomes a immature worm, which will then penetrate through the mucous layer to the serosal and then onto the liver surface (Kaya et al., 2011). Immature worms will continue to develop in the liver parenchymal cells and 
stimulate the sinusoids to absorb more nutrients. The immature worm will then reach the bile duct and develop into adult worms, resulting in the damage of the bile epithelial cells (Al-Megrin \& Al-Qahtani, 2016). This is also related to the number of fluke that invade the gallbladder. Acute fascioliasis can continue for several weeks to months and coincide with the migration of fluke through hepatic parenchyma cells. Alteration of liver enzymes level as observed in our study, may reflect the migration of the fluke. Liver cell death and damage to biliary epithelium will trigger the catabolism of ALT, AST and ALP, which can then be detected through blood serum (Senior, 2012).

In an experimental infection of Fasciola in rabbits, Jarujareet et al. (2018) observed that significant increase in serum ALT and AST appeared at $3 \mathrm{WPI}$ and reached a peak value at $6 \mathrm{WPI}$. The elevation of these enzymes relates to the liver inflammatory state and to tissue destruction provoked by parenchymal migration of young flukes during the early stages of fascioliasis. At $7 \mathrm{WPI}$, ALT and AST progressively returned to normal values probably due to the migration of juvenile flukes into the bile ducts and since the liver is an organ that regenerates itself comparatively quickly, ALT and AST returning to the normal level might reflect this observation (Jarujareet et al., 2018). In our study, we observed that the infected individual deer correspondingly had elevated ALT and AST. There is a possibility Timor deer might have different host response than that of the rabbits. On the other hand, there is also a possibility of recurrent infection by the parasite to constantly stimulate the host response.

Liver enzyme profiles is being used as a health indicator in disease surveillance and periodical monitoring of the well-being of Timor deer at the Surabaya Zoo and also by other deer breeders. The results of our study can provide the baseline data for health maintenance and its association with fascioliaisis in Timor deer. We concluded that detection of elevated levels of serum ALT, AST and ALP in Timor deer may be indicative of fascioliasis. Since the EPG showed a positive correlation with serum ALT, AST, and ALP levels, measurement of these enzymes may indicates the severity of the infection or parasitic burden. Our study showed that the Timor deer at Surabaya zoo were probably infected with only F. gigantica.

\section{ACKNOWLEDGMENTS}

We thank the government of Surabaya and Surabaya Zoo for allowing us to collect the deer samples and also Profs. Pudji Srianto, Fedik Abdul Rantam, Mufasirin, Suwarno and Muhammad Yunus of Faculty of Veterinary Medicine, Universitas Airlangga and also Suryanto (PSDKU Banyuwangi) for their support.

\section{Funding information}

This work was supported by the Institute of Research and Innovation, Universitas Airlangga and Faculty of Veterinary Medicine, Universitas Airlangga with grant number 487/ UN3.1.16/LT/16.

\section{Conflict of interest}

The authors declare that they have no conflict of interest.

\section{REFERENCES}

Al-Megrin, W.A. \& Al-Qahtani, W.S. (2016). Apoptotic gene expression in sheep hepatocytes during Fasciola hepatica infection (Fascioliasis). Biotechnology Journal International 12: 1-11. https://doi.org/10.9734/BBJ/2016/24186
Conceição, M.A.P., Durão, R.M., Costa, I.H. \& da Costa, J.M.C. (2002). Evaluation of a simple sedimentation method (modified McMaster) for diagnosis of bovine fascioliosis. Veterinary Parasitology 105: 337-343. https://doi.org/10.1016/ s0304-4017(02)00016-x

Edith, R., Godara, R., Sharma, R.L. \& Thilagar, M.B. (2010). Serum enzyme and hematological profile of Fasciola gigantica immunized and experimentally infected riverine buffaloes. Parasitology Research 106: 947-956. https://doi.org/10.1007/ s00436-010-1741-1

Hodzić, A., Zuko, A., Avdić, R., Alić, A., Omeragić, J. \& Jazić, A. (2013). Influence of Fasciola hepatica on serum biochemical parameters and vascular and biliary system of sheep liver. Iranian Journal of Parasitology 8: 92.

Hyder, M.A., Hasan, M. \& Mohieldein, A.H. (2013). Comparative levels of ALT, AST, ALP and GGT in liver associated diseases. European Journal of Experimental Biology 3: 280284.

Jarujareet, W., Taira, K. \& Ooi, H.K. (2018). Dynamics of liver enzymes in rabbits experimentally infected with Fasciola $\mathrm{sp}$. (Intermediate form from Japan). Journal of Veterinary Medical Science 80: 36-40. https://doi.org/10.1292/jvms.170315

Kaya, M., Beştaş, R. \& Çetin, S. (2011). Clinical presentation and management of Fasciola hepatica infection: singlecenter experience. World Journal of Gastroenterology 17: 4899-4904. https://doi.org/10.3748/wjg.v17.i44.4899

Kowalczyk, S.J., Czopowicz, M., Weber, C.N., Müller, E. \& Kaba, J. (2018). Accuracy of a diagnostic model based on serum biochemical parameters in detecting cows at an increased risk of chronic fascioliasis. Veterinary Parasitology 254: 15-20. https://doi.org/10.1016/j.vetpar.2018.02.038

Martins, R.F., Schmidt, A., Lenz, D., Wilting, A. \& Fickel, J. (2018). Human mediated introduction of introgressed deer across Wallace's line: Historical biogeography of Rusa unicolor and R. timorensis. Ecology and Evolution 8: 1465-1479. https://doi.org/10.1002/ece3.3754

Masyud, B., Wijaya, R. \& Santosa, I.B. (2007). Distribution, Population and Daily Activities of Timor Deer-Cervus timorensis, de Blainville 1822 in Bali Barat National Park. Media Konservasi 12: 1-6.

Ozel, B.D., Yagbasan, A. \& Karaman, A. (2013). An Uncommon Liver Mass and Hypereosinophilia: Acute Fascioliasis. Journal of Medical Cases 4: 785-788. https://doi.org/10.4021/ jmc1534w

Ozturhan, H., Emekdaş, G., Sezgin, O., Korkmaz, M. \& Altintaş, E. (2009). Seroepidemiology of Fasciola Hepatica in Mersin province and surrounding towns and the role of family history of the Fascioliasis in the transmission of the parasite. Turkish Journal of Gastroenterology 20: 198203. https://doi.org/10.4318/tjg.2009.0007

Park, G.J.H., Lin, B.P., Ngu, M.C., Jones, D.B. \& Katelaris, P.H. (2000). Aspartate aminotransferase: alanine aminotransferase ratio in chronic hepatitis $\mathrm{C}$ infection: is it a useful predictor of cirrhosis? Journal of Gastroenterology and Hepatology 15: 386-390. https://doi.org/10.1046/j.14401746.2000.02172.x

Robinson, M.W. \& Dalton, J.P. (2009). Zoonotic helminth infections with particular emphasis on fasciolosis and other trematodiases. Philosophical Transactions of the Royal Society B: Biological Science 364: 2763-2776. https://doi.org/ 10.1098/rstb.2009.0089

Senior, J.R. (2012). Alanine aminotransferase: a clinical and regulatory tool for detecting liver injury-past, present, and future. Clinical Pharmacology and Therapeutic 92: 332339. https://doi.org/10.1038/clpt.2012.108 
Sezgin, O., Altintas, E., Disibeyaz, S., Saritas, Ü. \& Sahin, B. (2004). Hepatobiliary fascioliasis: clinical and radiologic features and endoscopic management. Journal of Clinical Gastroenterology 38: 285-291. https://doi.org/10.1097/ 00004836-200403000-00017

Tejada, J.J. \& Punzalan, J.R.B. (2012). On the misuse of Slovin's formula. The Philippine Statistician 61: 129-136.

Tolan Jr, R.W. (2011). Fascioliasis due to Fasciola hepatica and Fasciola gigantica infection: an update on this 'neglected' neglected tropical disease. Laboratory Medicine 42: 107116. https://doi.org/10.1309/LMLFBB8PW4SAOYJI

Valero, M.A., Perez-Crespo, I., Periago, M.V., Khoubbane, M. \& Mas-Coma, S. (2009). Fluke egg characteristics for the diagnosis of human and animal fascioliasis by Fasciola hepatica and F. gigantica. Acta Tropica 111: 150-159. https:// doi.org/10.1016/j.actatropica.2009.04.005

Warne, R.T. (2014). A primer on Multivariate Analysis of Variance (MANOVA) for behavioral scientists. Practical Assessment, Research, and Evaluation 19: 17. 